\title{
Wavelet study of Dynamic Variations in Steel and Ironmaking Rest Gases. Potential effect on external use. 茨
}

\author{
Niklas Grip ${ }^{\mathrm{a}, *}$, Carl-Erik Grip ${ }^{\mathrm{b}}$, Leif Nilsson ${ }^{\mathrm{c}, 1}$ \\ ${ }^{a}$ Division of Mathematical Sciences, Luleå University of Technology, SE-97187 Luleå, \\ Sweden \\ ${ }^{b}$ Division of Energy Science, Lulea University of Technology, SE-971 87 Luleà, Sweden \\ ${ }^{c} S S A B$ EMEA, SE-971 88 Luleå, Sweden
}

\begin{abstract}
The surplus energy in rest gases are an important by-product in the steel plant in Luleå. The surplus is used in a local heat and power plant. Studies have been and are being carried out to find alternative use for the high calorific ones, especially the coke oven gas. A limitation could be fluctuations in gas availability. The scope of this work has been to study these fluctuations and estimate possible effects on the use. Logged data from one year of the steel plant have been used. A large part of the signals have non-periodic contents and/or irregularly distributed transients. A specific technique, wavelet analysis, has been used to handle that. For this reason the signals were analysed using wavelet analysis.

The study shows that there are important gas fluctuations that could influence external use, e.g., for fuel production and that the behavior was non periodic. In addition to variations in frequency and amplitudes, there are also different shapes of the fluctuations, e.g. a relatively high ratio of "spikes" that could be up-or downward to the final users. The fluctuations are of both short and long wavelength. Longer wavelengths could probably be parried by good production planning. For short wavelengths some kind of safety margin could be appropriate, perhaps in the range $0.75-1.5 \mathrm{kNm}^{3} / \mathrm{h}$. The existing buffer capacity in gasholders etc. is too small to cover the
\end{abstract}

\footnotetext{
*Corresponding author

Email addresses: Niklas.Grip@ltu.se (Niklas Grip ), Carl-Erik.Grip@ltu.se (Carl-Erik Grip ), Leif-L.Nilsson@ssab.com (Leif Nilsson )
} 
fluctuations. Good production planning can partly compensate low buffer capacity. An example from a period without gas holder is discussed. An additional objective was to test the wavelet technique for that environment. It showed to be well aimed to analyse the different types of intermittent and irregular variations with different waveforms that are abundant in the steel plant network.

Keywords: rest energy, frequency analysis, fuel conversion, energy systems for power generation, environment and climate change, wavelet analysis

\section{Introduction}

The ore based steel plant of SSAB EMEA in Luleå produces around 2.2 Mtonnes of steel every year. The steel is produced as slabs that are transported to the SSAB EMEA rolling mill in Borlänge, which produces high quality steel strips. A byproduct from the steel production is energyrich by-product gases. It is common practice to use those gases as fuels for reheating in the rolling mill. This is not possible in Lulea because of the distance to the rolling mill $(800 \mathrm{~km})$, so the gases have to be used locally. Presently, they are used in a local combined heat and power plant (CHP) to produce electricity and district heating (important close to the arctic circle). The structure of the gas net is shown in Figure 1. The sketch is superimposed on a plant drawing.

The coke oven plant produces coke by dry distillation of coal with coke oven gas (COG) as a byproduct. The gas is high calorific with a lower heat value in the range of $18 \mathrm{MJ} / \mathrm{Nm}^{3}$. Part of the gas is used to heat the coke oven battery. The coke oven plant has an internal gas holder with a volume of $6000 \mathrm{~m}^{3}$. It is working as safety storage to ensure that there is always fuel in the battery, and it is not used as a buffer for the delivery to outside units. Because of the distance there is no connection between the coke oven plant and the steam network in the rest of the plant. Instead a local boiler, fired with coke oven gas is used to supply the gas cleaning system with steam and heat. In 2006 the total production of coke oven gas was 4450 TJ. Of this 2250 was used to heat the battery and 550 for steam generation. Of the remaining coke oven gas 460 TJ was used at the blast furnace mainly in the hot stoves for blast heating. $310 \mathrm{TJ}$ was used in the steel plant area, mainly for drying and heating of ladles and torpedo cars. 470 TJ was exported to the lime furnace and the heat and power plant. 


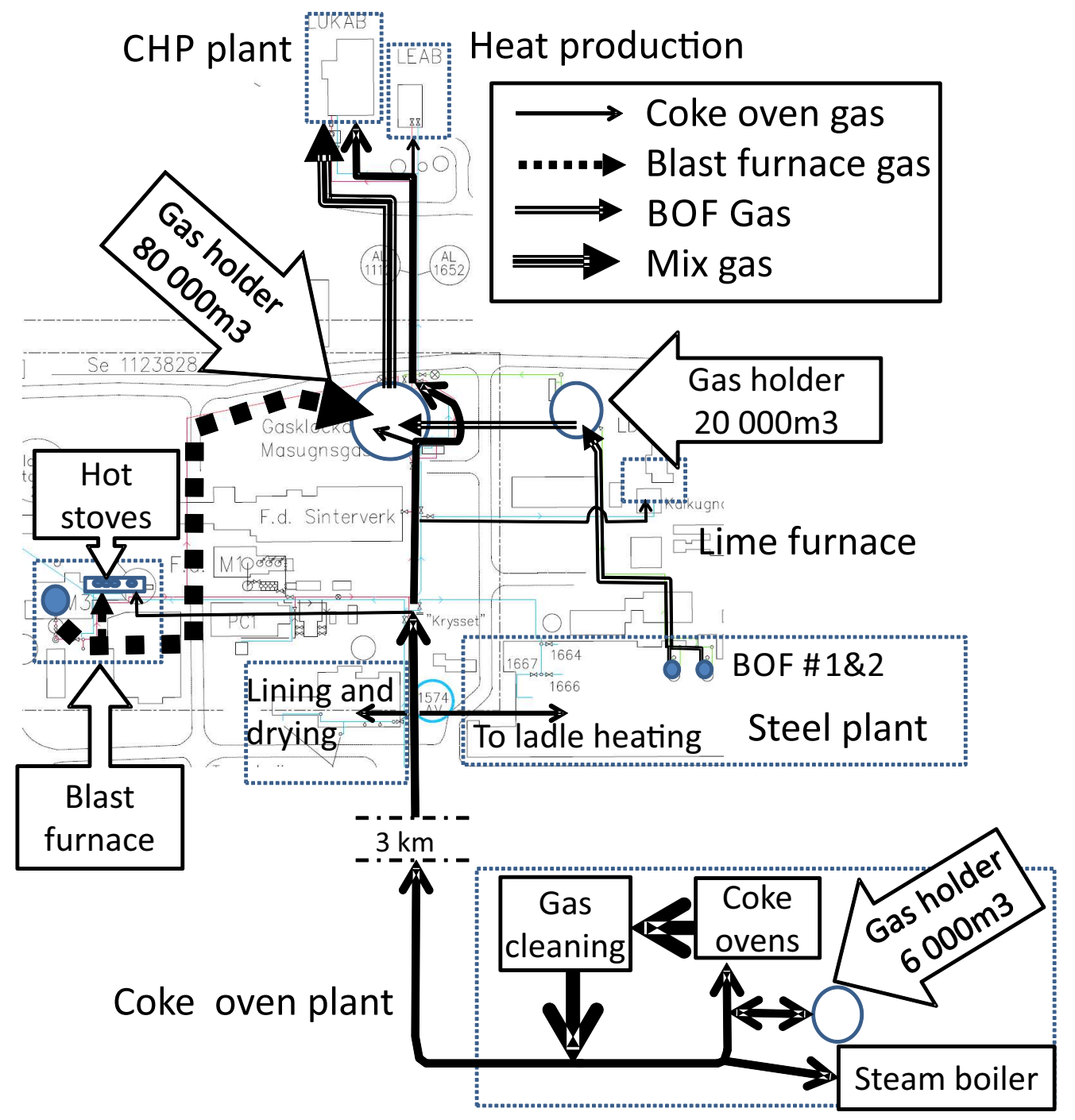

Figure 1: Gas net of system Coke plant - Blast furnace - Steel plant CHP. (Superimposed on plant drawing.)

The blast furnace produces liquid hot metal with blast furnace (BF) gas as a byproduct. The gas is low calorific with a lower heat value in the range of 2.7-3 MJ $/ \mathrm{Nm}^{3}$. Part of it is mixed with coke oven gas (to get sufficient heat value) and used in the hot stoves. The rest is sent to the mixgas holder (volume $80000 \mathrm{~m}^{3}$ ) for export to the CHP plant.

The basic oxygen furnace (BOF) converters in the steel plant produce 


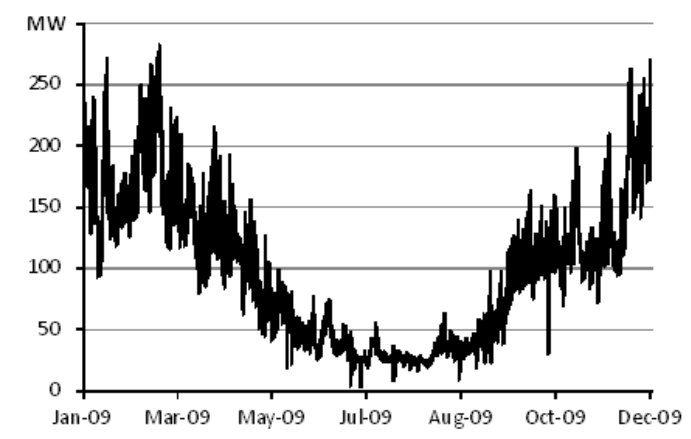

Figure 2: Consumption of district heating energy 2009.

steel with BOF gas. The gas is relatively high calorific with a lower heat value in the range of 6-8 $\mathrm{MJ} / \mathrm{Nm}^{3}$. The two converters operate independently of each other. They produce gas around 15 minutes per heat with a tap-to tap sequence in the range of 40-45 minutes per heat. A gasholder with a volume of $20000 \mathrm{~m}^{3}$ is installed as a buffer, as the gas is produced batch wise but used continuously. The gas from the BOF gasholder is sent to the mix gas holder, where it is mixed with $\mathrm{BF}$ gas to get a mix with increased heat value. If necessary the heat value is further increased by addition of some coke oven gas into the gasholder. The remaining the coke oven gas (after export to the lime furnace) is sent directly to the coke oven plant and used in special coke gas burners. Figure 1 also shows a heating plant close to the CHP, which is used as reserve capacity in case of very high consumption of district heating.

The heat and power plant supports a district heating net, which in principle covers the need of heating for all the housing in the city of Lulea. The CHP plant is owned and run in common by the SSAB steel plant and the community of Luleå. It is built so that it can work as a counter pressure plant delivering both heat and electrical power, as a cold condense plant delivering only electrical power or as a combination between these modes. The delivery of heating is guaranteed by the owners. The consumption of district heating during 2009 is shown in Figure 2. During the main part of the year, there is a surplus which is used to produce electricity, by running the CHP plant with a combination of cold condense and counter pressure mode. An exception is the coldest part of the year (usually mid- February) when a practice with $100 \%$ counter pressure, and sometimes also an addition of oil is needed to cover the need of heating. Discussions on a possible production increase could however increase the surplus. 
The gases are of different quality. The blast furnace gas has a relatively low heat value, usually around $3 \mathrm{MJ} / \mathrm{Nm}^{3}$. It is available in large amounts and the major fuel for the heat and power plant and the hot stoves. The BOF gas is usually in the range of $6-8$ and the COG is around $18 \mathrm{MJ} / \mathrm{Nm}^{3}$. These two gases are high-calorific. Presently there is no difference in valuation of high and low gas as marginal gases are used as fuel in the CHP, where they in principle are interchangeable.

It would be of great interest to use the surplus of high calorific gases for more high-qualified uses. One solution could be to use them to produce liquid car fuel [1]. The technical and economical possibility to do this has been studied in a project co-financed by the Swedish Energy Agency, SSAB EMEA, Grontmij AB and Nordlight AB. The project included studies of coke oven gas alone as well as its use in combination with BOF gas and synthetic gas from gasification of biomass $[2,3]$. The work included studies of possible technologies, as well as technical and economical calculations and optimizations. The optimizations were based on process integration using the tool reMIND, which had been developed together with Linköping University [4] and is extensively used with Swedish steel and mining industry [5-7] as well as abroad $[8,9]$. It was also decided to base the study on production data from the year 2006. The reason was that this was a year with full production and conditions corresponding to the expected practice. The efficiency and economy of such a plant is, however, influenced not only by the mean values of the gas availability, but also of long and short period fluctuations in the gas supply. These all have variations in amplitude and frequency and cannot be expected to be in phase with simultaneous variation at the potential users. Large variations could make it necessary to create a safety margin by sending more of the surplus gases to less valuable use as CHP fuel. For this reason it was decided to include a study of the fluctuations in the coke oven gas flow and use.

A commonly used mathematical tool for studying the frequency contents of a measured signal is Fourier analysis. It is based on a decomposition of the signal into a sum of sine and cosine oscillations of different frequency and amplitude. This makes it well suited for analysing periodic variations, but it does have shortcomings when it comes to signals with non-periodic contents or irregularly distributed transients. This is partly confirmed by previous experiences at SSAB where some fluctuations of higher frequencies tended to be extinguished if the Fourier study was extended to a longer period $[2$, Section 3.5]. A possibility to make the studies relevant for not fully periodic 
cases is to use wavelet analysis. This method decomposes the signal into a sum of short wave packages, "wavelets", instead of continuous sinus curves (see more detailed description in Section 2.2). A study has been carried out to evaluate the coke gas behaviour with wavelet analysis as the main tool. The purpose was to analyse and describe the variations in the coke oven gas flow and to estimate their effect on a potential external gas user. An additional purpose was to investigate the usability of the wavelet tool in that environment. It was agreed that this activity should be based on the same time period as the one that was used for rest of the main study (2006).

The scope of this paper is to describe that study, the results so far and their possible use.

\section{Material and methods}

We describe the collection of data in Section 2.1 and the wavelet analysis tools used on these data in Section 2.2.

\subsection{Collection of data}

The SSAB steel plant has an extensive database which could be used for the study. Almost all process data are logged and stored as one minute values as well as hourly, daily, weekly monthly and yearly means. To avoid overloading the one minute values are kept for one year and then erased. The other values are kept, in principle for ever. A data base was prepared from hourly data logged during 2006 from SSAB EMEA in Luleå. It was based on hourly data as one minute data were not available for that period.

\subsection{Discrete wavelet analysis}

Discrete wavelet analysis is based on a decomposition of the signal into a sum of short wave packages, wavelets, instead of sinus curves (see Figure 3). This makes it a suitable tool for "catching" physical phenomena in the measurements that are local in time, irregular, or periodic but with dominating frequencies that are changing during the measurements. Discrete wavelet analysis can be used for decomposing measurements into signal components that show the behavior of the measured values at different time scales. Contrary to Fourier analysis, which decomposes a signal into periodic signal components of different frequencies, wavelet analysis has the advantage that it is local in time. This is achieved by decomposing the measured signal into a sum of building blocks (basis functions) that each has a very well-defined 

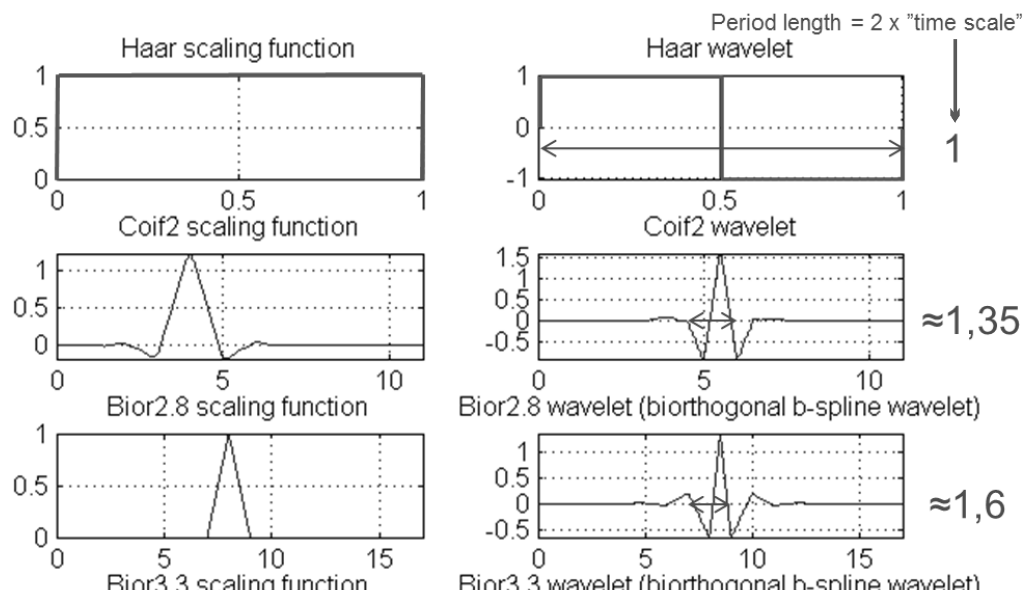

Bior2.8 wavelet (biorthogonal b-spline wavelet)
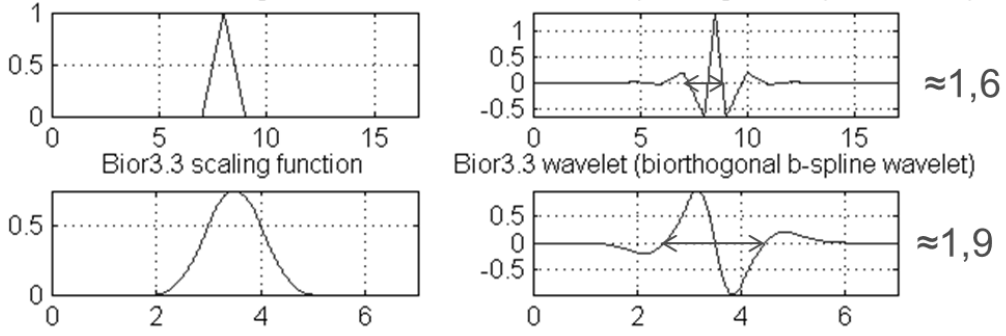

Bior3.3 wavelet (biorthogonal b-spline wavelet)

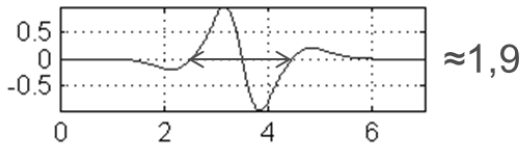

Figure 3: A few examples of wavelets available in the Matlab Wavelet Toolbox. The time scales indicated in the figures in Section 3.2 correspond to half the period length indicated in red in the right-hand plots.

location and contain variations on a very well defined time scale. For example, the topmost two plots in Figure 3 show the box-shaped Haar scaling function $\varphi$ and Haar wavelet $\psi$. Integer translates $\varphi(t-n)$ of the Haar scaling function, each multiplied with some constant $a_{n}$, can be used to approximate a signal by mean values on intervals of length 1 (time scale 1 ). For a finer approximation with mean values on intervals of length $\frac{1}{2}$ (time scale $\frac{1}{2}$ ), one can add integer translates $\psi(t-m)$ of the Haar wavelet, each multiplied with some constant $b_{0, m}$. Next, by shrinking the Haar wavelet to half its original length, one can add a layer of smaller building blocks $\psi(2 t-m)$ catching details in the signal that have time scale $\frac{1}{4}$. By proceeding in the same way, one can build an arbitrarily fine-scale approximation of the signal, say, with finest time scale $2^{-J-1}$ :

$$
s(t)=\sum_{n=-\infty}^{\infty} a_{n} \varphi(t-n)+\sum_{j=0}^{J} \sum_{m=-\infty}^{\infty} b_{j, m} \psi\left(2^{j} t-m\right)
$$

The Haar wavelet is well localized in time and has certain mathematical properties (orthogonality and completeness) that guarantee that any square integrable function can be arbitrarily well approximated by choosing large 

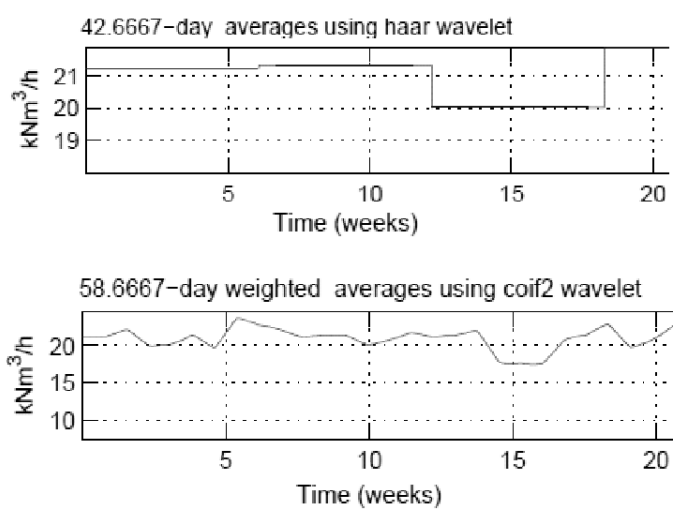

37.3333-day weighted averages using bior 3.3 wavelet

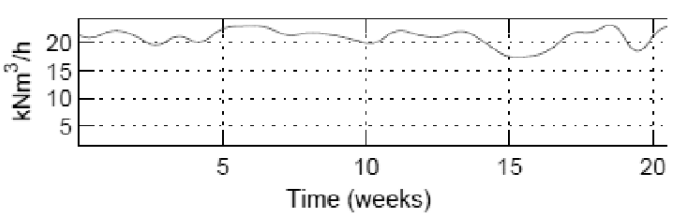

Figure 4: Comparison of curves created using Haar wavelets, Coiflets of order 2 and biorthogonal wavelets respectively. The curves correspond to the first 20 weeks of 2006 .

enough $J$ in (1), with uniquely determined coefficients $a_{n}, b_{j, m}$ and a fast algorithm for computing them. The main disadvantage of the Haar wavelet is bad frequency localization, or in other words that large $J$ is needed for good piecewise constant approximations of smooth functions. It is shown in [1012] and most wavelet textbooks how to construct smoother "building blocks" (wavelets) that share all the mentioned good properties of the Haar wavelet, such as the wavelets and corresponding time scales depicted in Figure 3.

Since different time scales are represented separately, by differently sized wavelets (different $j$ ), the signal can easily be decomposed into a sum of signals showing different time scales. Figure 4 shows a comparison of the similar (weighted) time averages of a signal computed with different wavelets from Figure 3. The topmost plot shows a Haar wavelet approximation of medium length variations. The lowermost two plots in Figure 4, show similar time scale approximations with smoother wavelets, which typically give better approximations depending on how smooth the analysed signal is. For the results in Section 3, we used the Coif 2 wavelet for reasons explained in Section $4.1-4.2$. 


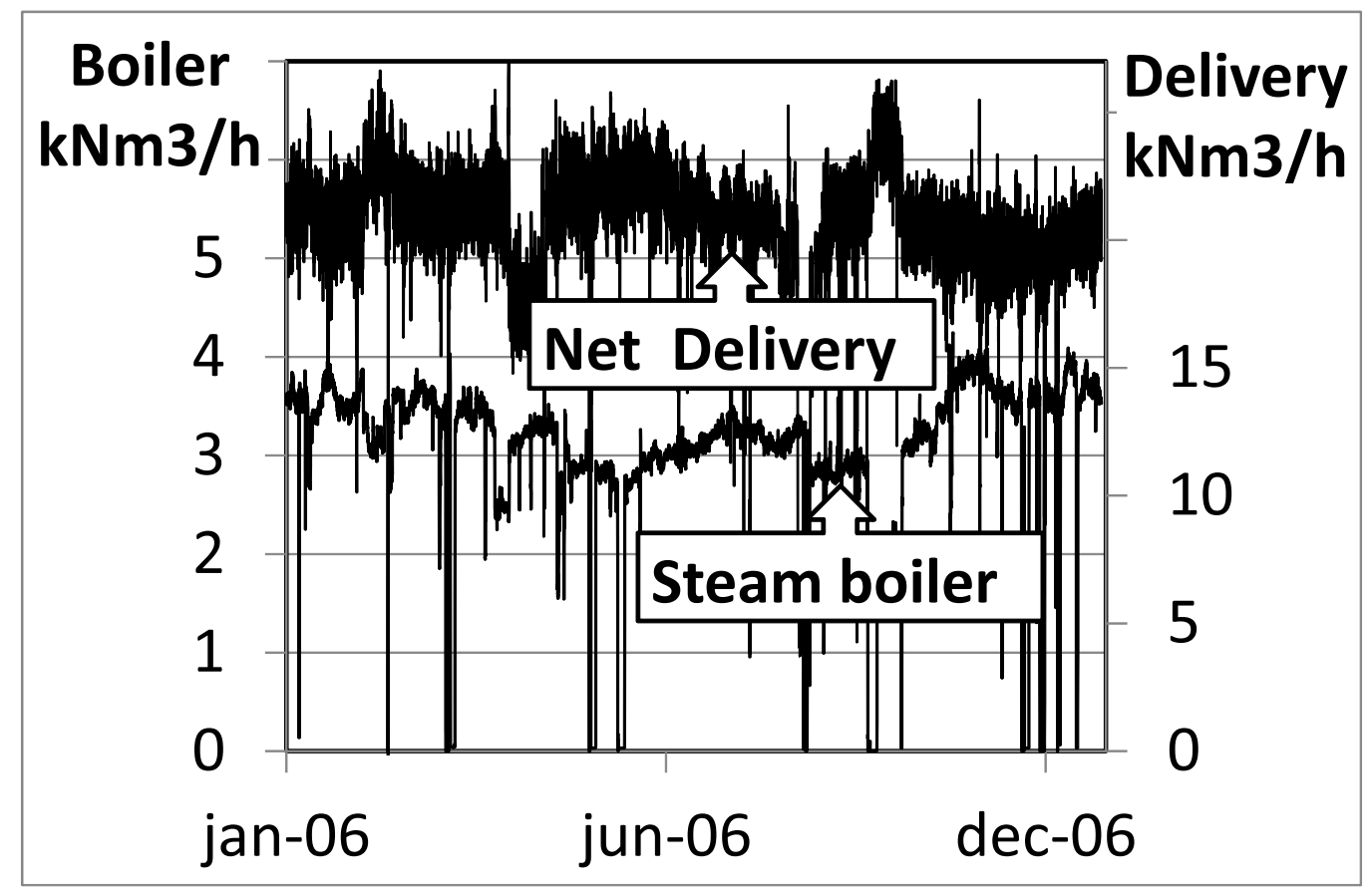

Figure 5: Hourly data showing volume flow of COG at the coke oven plant during 2006, net delivery and boiler consumption.

\section{Results}

\subsection{General analysis of hourly data}

The data logging of the plant uses minute, hourly, weekly and monthly means. The minute means are however saved only for a limited time, roughly 54 weeks, and thus not available for 2006. The study of data from 2006 is therefore based on logged hourly means. The data were plotted vs time and the plots were studied. Some examples are shown here.

The net delivery of the coke oven gas during 2006 from the coke oven plant is shown in Figure 5. The consumption of gas for the internal boiler is shown in the same diagram. More parameters could not be shown because of readability. A comparison of the curves show a mirror effect: Periods of higher consumption seem to correspond to a decreased net delivery and vice versa. This is expected as the net delivery is measured after taking away gas for the steam boiler. There are both long term variations of time scale several days, perhaps weeks, overlapped with short fluctuations. 


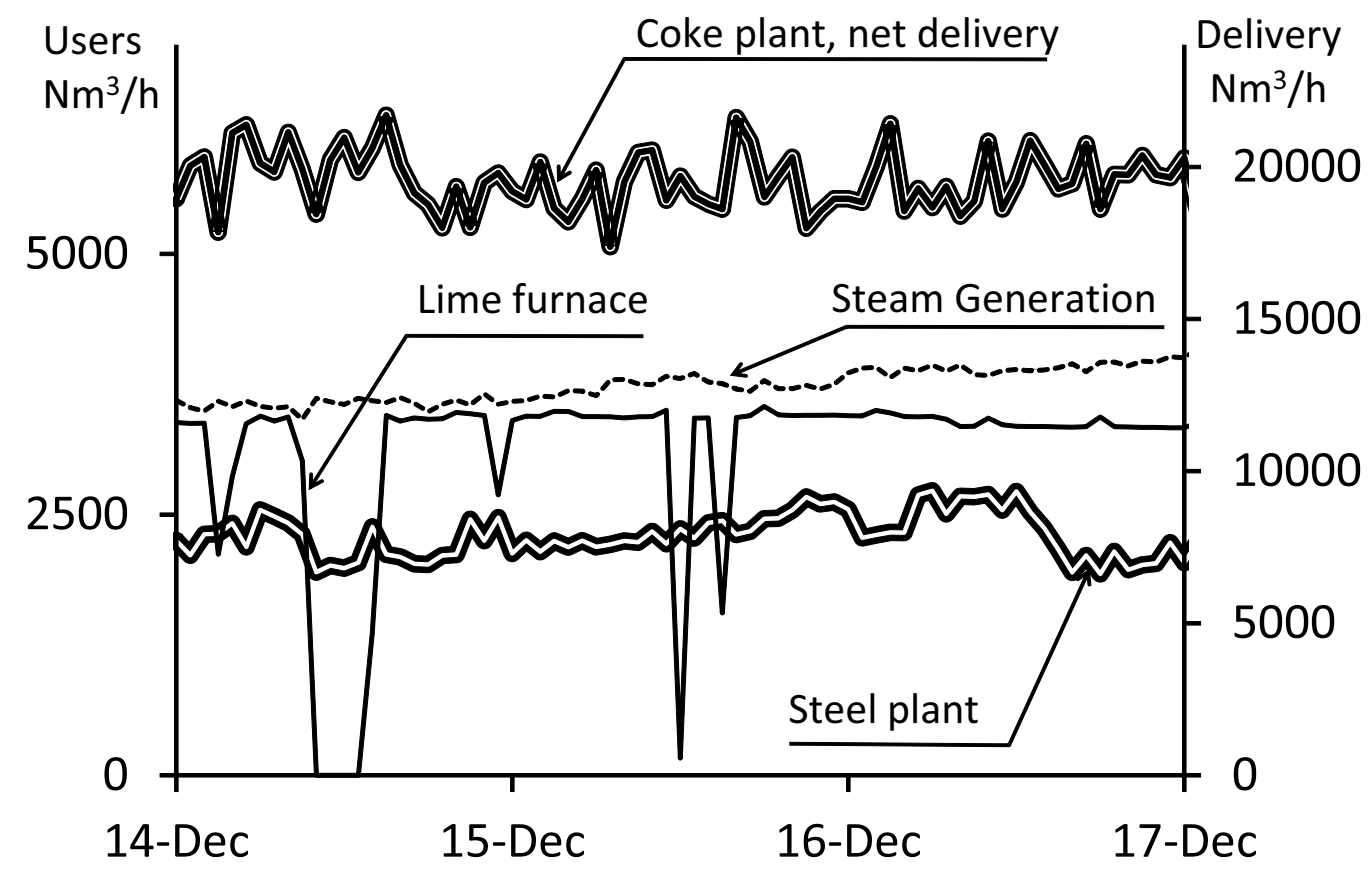

Figure 6: A 2006 three-day plot of the coke plant COG delivery and some consumers. From top to bottom: a) net delivery, b) consumption for coke oven plant steam production, c) consumption for full lime production and $d$ ) consumption for the steel plant ladle heating.

However, the short variations have a bad visibility in that timescale. They appear more clearly in Figure 6, which shows a shorter time period. In that diagram it was also possible to show two more COG consumers with acceptable visibility: the lime furnace and the steel plant (mainly ladle heating). For the lime furnace a major revamping was finished in the third quarter of 2006, leading to a remarkable increase in production. The diagram in Figure 6 shows a period in the late part of the year when full production was reached again. A relatively short period has been chosen to simplify a readable comparison.

The "mirror effect" for the steam generation is less visible in the short time range. Both steam generation and the ladle heating in the steel plant seem to have relatively regular fluctuations of a relatively short wavelength.

The curve form for the lime furnace is different. During the most of the time there is a relatively constant consumption with small variations, which 

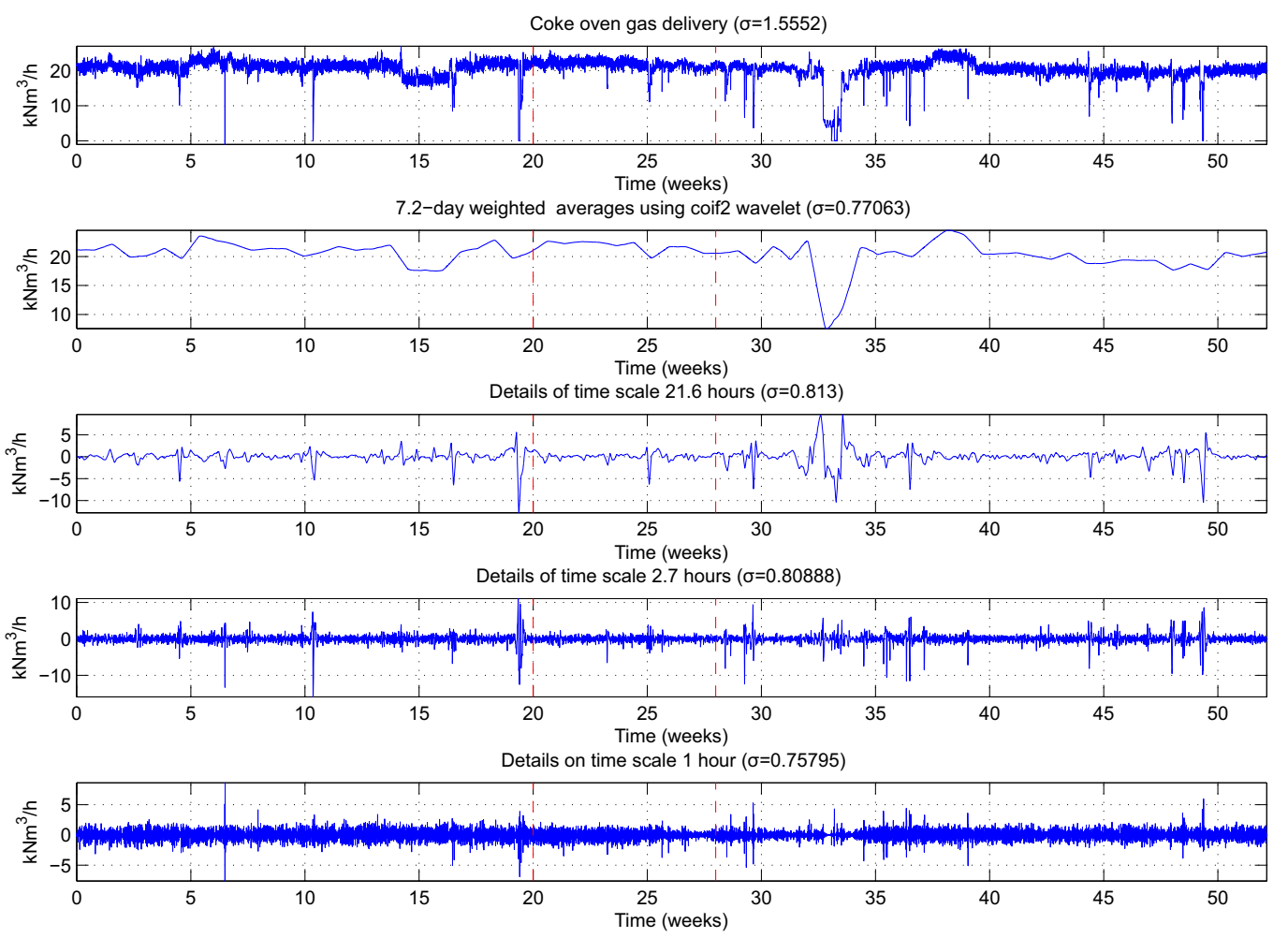

Figure 7: Coif 2 wavelet decomposition of coke oven gas delivered from the coke oven plant during 2006. (Same as the topmost curve in Figure 5 and 6.)

is interrupted by stops with zero use at irregular intervals. Such irregularly occurring transients makes these signals less well suited for Fourier analysis, which primarily looks for periodic behaviours.

\subsection{Wavelet analysis of hourly data}

Figure 7 shows an example of variations in gas volume flow and the components that were obtained by means of a Coif 2 wavelet decomposition. The upper plot shows the COG delivered from the coke oven plant (same as the topmost curve is Figure 5). The next three plots show decomposed components with three time scales: 7.2 days, 21.6 hours and 2.4 hours. The lowermost plot shows the hourly variations that remain after subtracting the more coarse scale fluctuations in subplots $2-4$. Consequently, the topmost original signal equals the sum of the other four signal components plotted in Figure 7. 

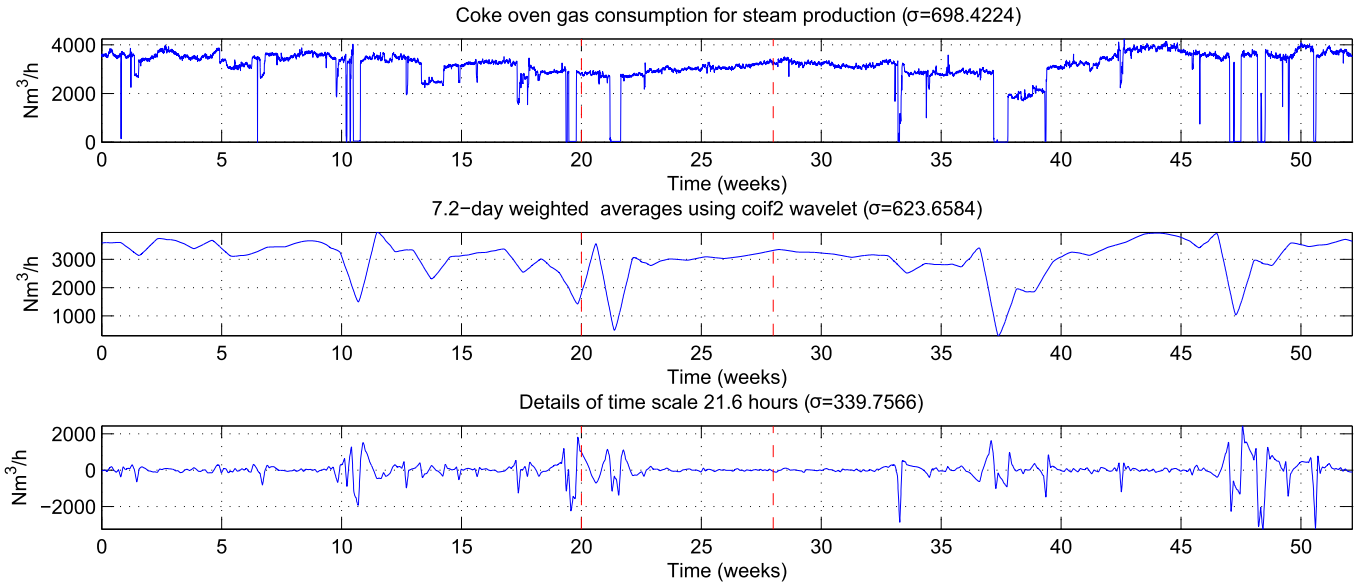

Details of time scale 2.7 hours $(\sigma=146.251)$
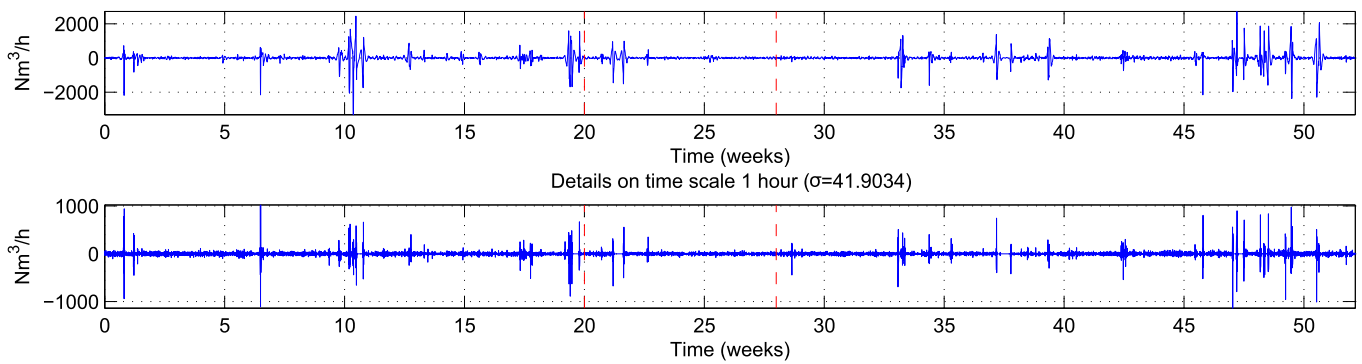

Figure 8: Consumption of COG for steam production at the coke oven plant: decomposition using Coif 2 wavelets. (Same as the steam generation curve in Figure 5 and 6.)

Similar diagrams for the other variables was analyzed and compared with the gas delivery curve. One of them, the gas consumption at the steam boiler is shown in Figure 8. Some of the instabilities in that figure are also visible in Figure 7. The connection between them is that the net delivery in Figure 7 is the gas that remains after the consumption of gas in the steam boiler. In addition to the previously mentioned mirror effect it can be noticed that the disturbances in the gas use at the boiler is reflected as irregularities in the delivery. This is the case mainly for shorter wavelengths.

The effect of the variations is not $100 \%$ comparable just by watching the diagrams. For this reason also the standard deviation of the variables for each timescale was calculated. This makes it possible to make a numerical comparison. This was made for week $20-28$, which was a period without big stops. The standard deviations $(\sigma)$ during week $20-28$ are printed in the sub-plot titles in Figure 7 and 8. 


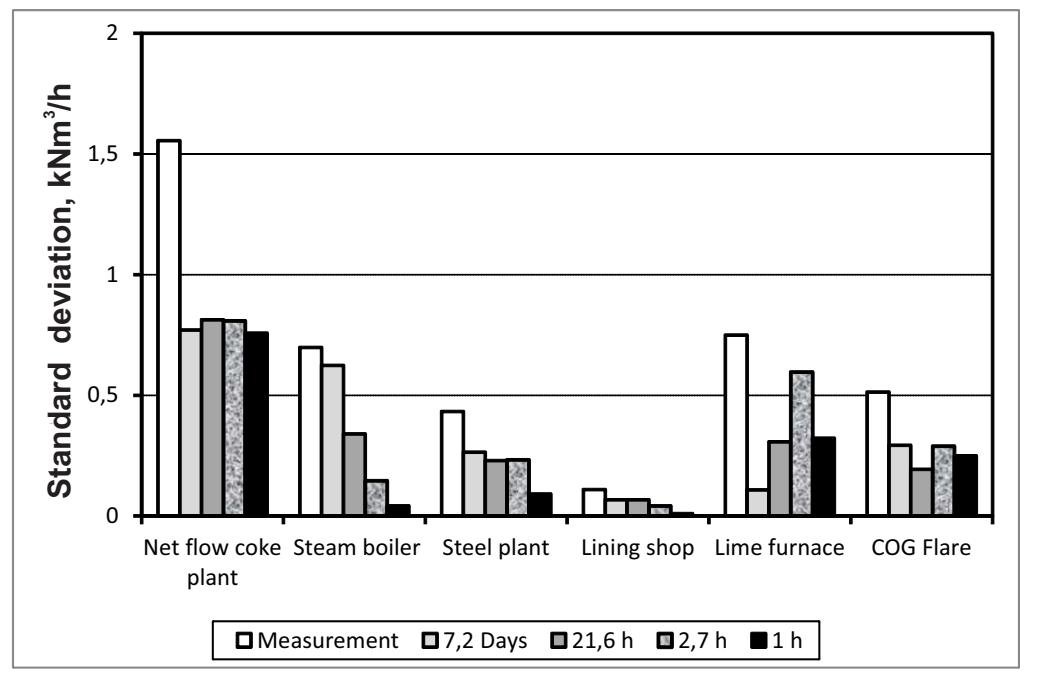

Figure 9: Fluctuations of different wavelengths expressed as standard deviation during week 22-28, (week 39.5-47.5 for the lime furnace).

The fluctuations for some important flows are summarized in Figure 9. The diagram shows the standard deviations calculated for each time scale as described in Figure 7 and 8.

For the net delivery, the variations are of similar magnitude for the different time scales. The one hour variations are slightly higher. For the steam boiler the main fluctuations seem to be a week or longer. The steel plant in principle shows an equal mix of long and short variations. Lime furnace and flaring have a relatively high amount of short fluctuations. (The reason for the different time period for the lime furnace is that it was revamped in the summer and was running with the present capacity from the autumn).

\section{Discussion}

\subsection{Effect of different waveforms}

The first studies (not shown in this paper) were made using the Bior 3.3 wavelet. This one uses a wavelet formed as a sinus wave with equal distribution of upwards and downwards deviations. This is OK if the variations in the tested parameter is a mix of up-and down variations. The result could be expected to be less successful if the analysed parameter has a quite different waveform. One example is the lime furnace where the main deviations are downward "spikes". For this reason that gas flow was analysed using another 


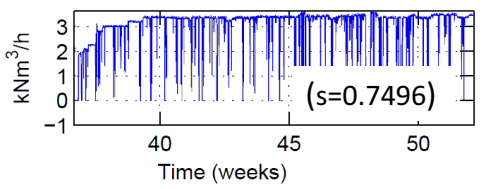

Measured

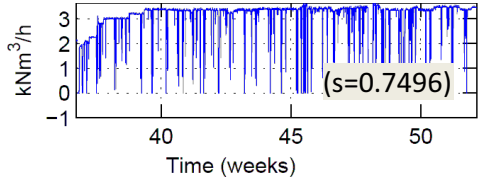

Measured

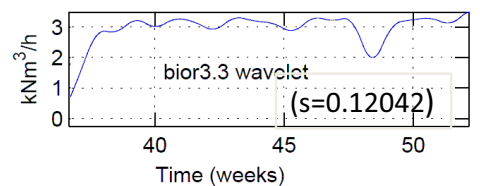

5.3 days

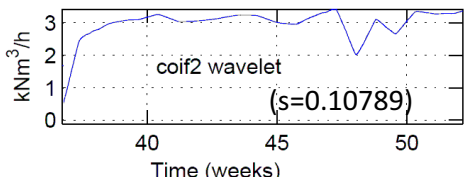

7.2days

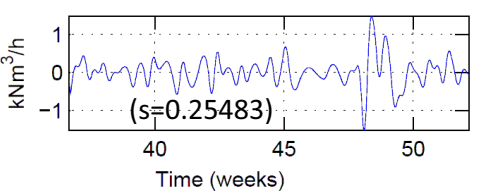

1.3 days

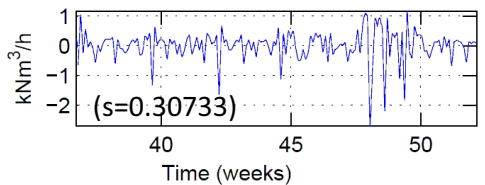

0.9 days

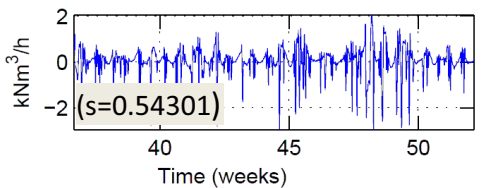

4 hours

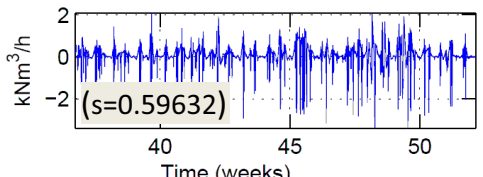

2.7 hours

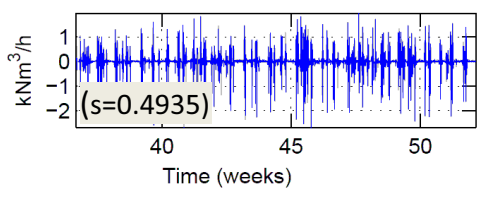

a) Bior 3.3 wavelet

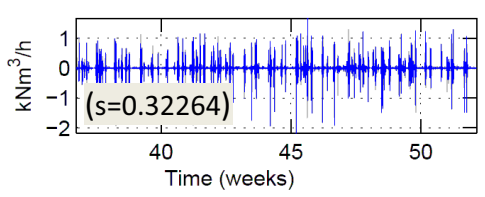

1 hour

Figure 10: Comparison of two types of wavelets for the COG flow to the lime furnace.

wavelet, coif2, which can be characterised as a vertical short "spike" which is balanced by two longer opposite waves of low height (see Figure 3). The results are compared in Figure 10. The diagrams shows an analysis of the lime furnace for the period after the revamp with the bior 3.3 analysis on the left hand side and coif 2 on the right hand side. The figure indicates that the coif2 analyses catches most of the downward deviations in the 4 hours and 1.3 days area and leaves a remainder of symmetric smaller fluctuations in the 1 hour area. This agrees comparably well with the real picture in Figure 6. The Bior 3.3 on the other hand misses several of these fluctuations. The difference is also visible in the calculated standard deviations, the Coif 2 analysis shows a lower standard deviation for the lower frequencies and a higher one for the higher. This shows that an analysis with one type of wavelet and a discussion on standard deviations is not sufficient for a full 
analysis. However, even if the standard deviations in Figure 10 differ, they are roughly of a similar magnitude independent of the chosen method. This indicates that a general analysis like in Section 3.1 can be useful as a preliminary scanning, but that it should be followed by studies with other tools, e.g., different waveforms and detailed manual studies.

\subsection{Choice of wavelet form}

Several waveforms are available as building blocks, see Figure 3. From Figure 10 it can be concluded that it is important to choose the right one, in order to get a comparison of high quality. All the logged COG flows were evaluated using Haar, Bior 2.8, Bior 3.3 and Coif 2 and plotted and represented like in Figure 7 and 8. To find a proper comparison criteria look at Figure 7, 8 and 10 above. The top diagram is the original measurement, in curve 2, 3 and 4 fluctuations of decreasing wavelength have been calculated and subtracted from the remaining data. The lowest curve (1 hour) shows the remaining fluctuation that could not be explained by the fluctuations that were found in curve 2-4. Thus the standard deviation of that curve is a measure of the quality of the analysis. A low value indicates a higher degree of explanation. In Figure 11, the different wavelet types are compared with that standard deviation as a criteria. The values for different flows are over a wide range of magnitude. A logarithmic scale was chosen to make the differences more readable. The coif 2 wavelet seems to show the lowest remaining fluctuation and thus the highest degree of explanation for all the studied flows. It seems to be the best one to analyse the types of flows that are studied here. It has been used in the comparisons in this study.

An additional conclusion is that asymmetric "spikes" seems to be an important contribution for all the logged flows.

\subsection{Some considerations on long and short fluctuations}

The study has shown that there are both long and short term fluctuations both in the delivered coke oven gas and at the present users of coke oven gas. A closer look at Figure 9 reveals that the total gas delivery has a rather high frequency of short wave fluctuations, whereas some user units have a higher proportion of more slow variation. Also the flaring, which can be seen as an indicator of system instability shows a relatively high proportion of short term variations.

If the longer term variations are predictable they can probably be compensated by a good production planning and control system. This decreases 


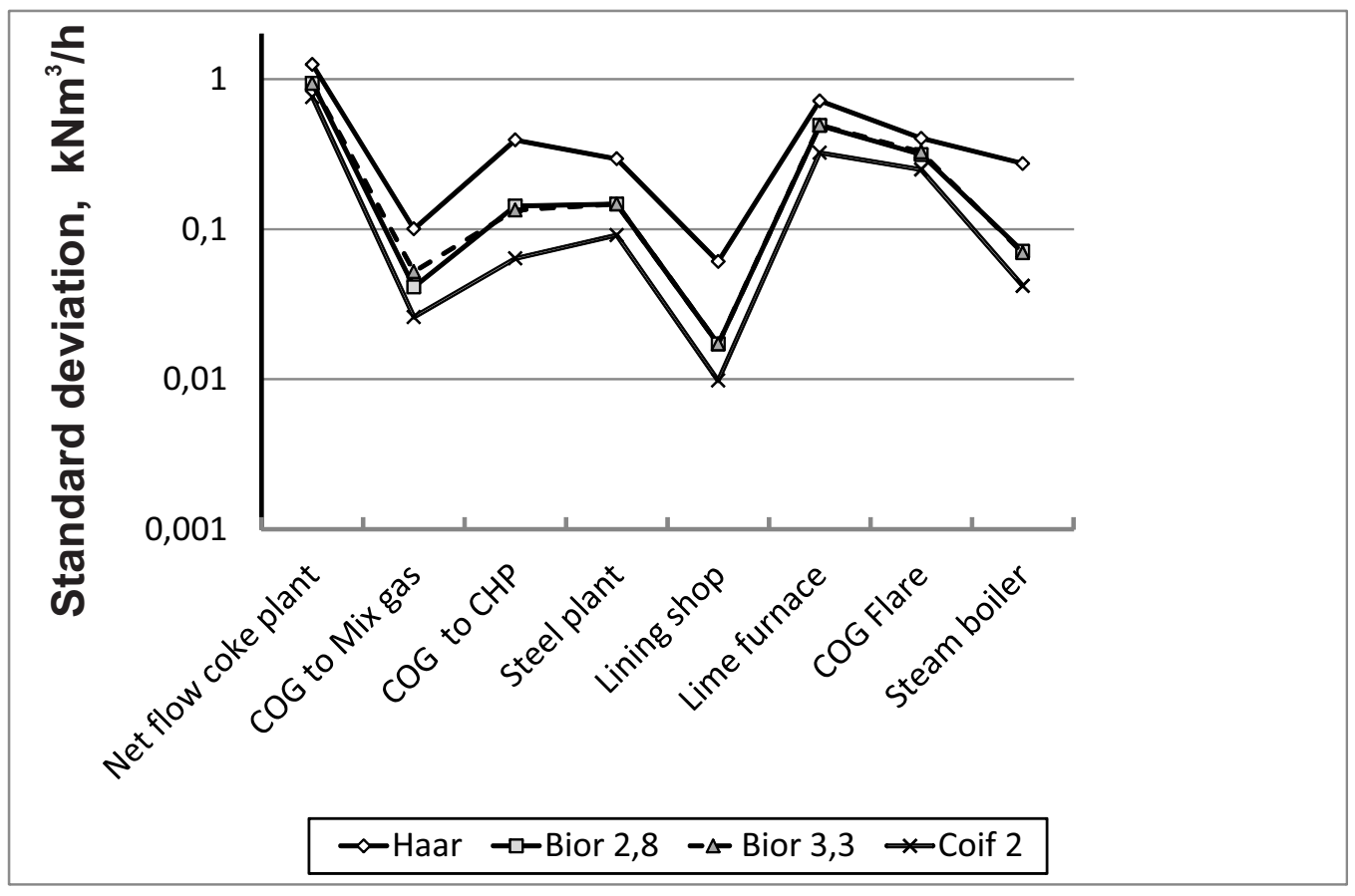

Figure 11: Comparison of different wavelet types. The diagram shows the standard deviation of the unexplained fluctuations in the $1 \mathrm{~h}$ remainder.

their impact somewhat. The purpose of the work on gas transients was to study if there are major variations in the gas flow that could influence the deliveries to a potential producer of methanol or other liquid fuels. In principle, a chemical factory can be expected to need a delivery with predictable and relatively constant flow and quality. If there are variations, part of that flow would have to be stored as a safety margin and sent to less valuable use. The fluctuations can be of importance the gas is to be used for production in an external unit, e.g. a fuel factory. In case of extensive fluctuations in gas, the factory management will probably have to add a safety factor on the gas rate, with the effect that part of it cannot be used for the right purpose. For symmetric fluctuations the standard deviation indicate on the safety factor that is needed. The exact value depends on the application, 2-3 standard deviations could be a good guess. The shorter variations can be more difficult to compensate in a control system. Instead they could be necessary to compensate with a safety margin. According to Figure 9 the short term fluctuations in net delivery from the coke-oven plant have a fluctuation cor- 
responding to a standard deviation of approximately $0.75 \mathrm{kNm}^{3}$ per hour. If a safety margin of two sigma is employed, it might be needed to reserve 1.5 $\mathrm{kNm}^{3}$ per hour for other use. One solution could be buffer capacities in the system, i.e. gas holders.

The fluctuations shown for the lime furnace are less dangerous. The stops do not lead to any lack of gas; instead there are short peaks in availability that are lost either to less profitable use or to flaring. i.e., the subsequent users get a gift that they cannot use but no disturbances.

\subsection{Interaction with gasholders}

Presently there are three gasholders in the system, one for COG at the coke plant, one for BOF gas at the BOF plant and one for the export gas to the CHP plant.

The coke oven gas holder has a volume of $6000 \mathrm{~m}^{3}$. This corresponds to around 18 minutes with a flow of $20000 \mathrm{Nm}^{3} /$ h. However, the COG holder at the coking plant is only used as safety storage to ensure that there is always gas for the under firing. It has always to be well filled to ensure underfiring in case of disturbances. Thus it cannot be used as a buffer for the delivery to other units. Likewise, the BOF gas holder is used as an internal buffer for the BOF plant, to compensate for the batch-wise delivery of gas from the converters. The effect of the buffer is insufficient if both converters are blown simultaneously and in that case some BOF gas has to be flared. This happens and cannot be avoided as the BOF planning has to be controlled by the need of steel at the continuous casters. The capacity is briefly exact if they are blown alternating.

The export gases from BOF and BF are mixed in the mix gas holder. That gas holder has a volume corresponding to 15-20 min of delivery. Generally there is a buffer on the total gas delivery, but no specific buffer for delivered COG. The BOF gas is used by the CHP plant both as a general fuel and as a mean to increase the heat value. The flow from the BOF gas holder and COG net to the mix gas holder is controlled by the CHP operators to ensure that purpose. With this practice the mix gas holder can only give a marginal buffer to BF gas and COG variations. (The COG can be sent directly or be sent through the mix gas holder and arrive 15-20 minutes later.)

A possibility to study the importance of the gas holders occurred in 1994 when the export gas holder was damaged by an accident and was inoperative until next summer. The failure happened in the summer of 1994 and the construction of a new holder started. The upper half of the new holder was 


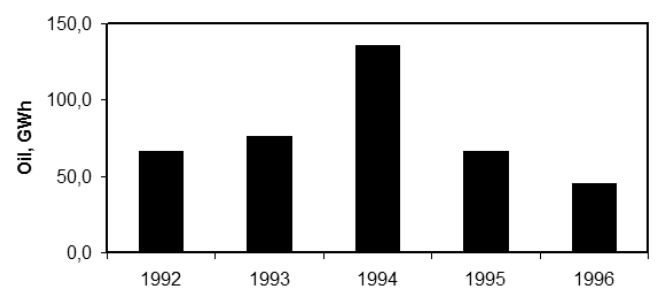

Figure 12: Gasholder failure 1994: Effect on oil consumption in the heat and power plant.

used provisionally during the winter, and in the spring the new holder was assembled and put in production. Also extra effort was made to fortify the manual gas control during the period. The CHP plant is fired with gas, but oil is used if the gas is not sufficient. Figure 12 shows the amount of oil that was used before 1994 and the years before and after. A heavy increase in oil consumption is seen in 1994 after the incident, and then it decreases again. It should however, be noted that the problem remained during spring 1995 until the new holder was put in production. Thus an increased consumption could be expected also during that year, but this did not occur. The reason is most likely the increased effort on resources and routines for gas control. One important conclusion is that a good production control could be as important as the buffer capacity.

A general question for future research (also for other process industries) could be if it is possible to include wavelet methods or results in production control algorithms.

\subsection{Measures to ensure delivery}

In the present system, excess COG is simply sent to the CHP and used together with BF gas. The driving force to decrease fluctuations will probably be higher if it is sent as a qualified product to a fuel producer. A primary measure could be a further development of the gas control system. A specific gas holder could be interesting but is expensive. Mixing stations to ensure that each user get the heat value they need, neither more nor less, could improve the availability of the high value gases (BOF and COG).

\subsection{Study of data with a one minute sampling rate}

The results in Section 3 were based on logged hourly data. It should be noted, that several processes can be expected to have fluctuations shorter 
than one hour. As example, the delivery from the coke-oven plant is influenced by emptying in sequences of 10 or 11 furnace chambers with an interval of approximately 15 minutes and around 1 hour between the sequences, change of gas flow direction in the lime furnace and the $\frac{1}{2}$-hour Cowper sequences etc. Thus, a one minute sampling rate is preferable whenever available. For this reason a tentative study was carried out with a limited amount of one minute data that were sampled one year back from the sampling time. Figure 13 covers a 19 hour interval of the logged data of net COG delivery (same variable as in Figure 7). The 15 minute time scale variations mentioned above are clearly visible.

\subsection{Wavelet Method: continuous type of representation}

The studies in Figure 7, 8 and 10 were made using fixed wavelength shapes that remain constant over time. In reality also the frequencies tend to change gradually with time. This could be of interest to study also these trends. This can be made using another tool for finding periodically repeating signal components: The continuous wavelet transform, which can be used to plot how the frequency contents varies with time, as shown in Figure 14 for COG delivery measurements. (See, for instance, [12] for a mathematical background.) A bright horizontal line indicates a detected periodic signal

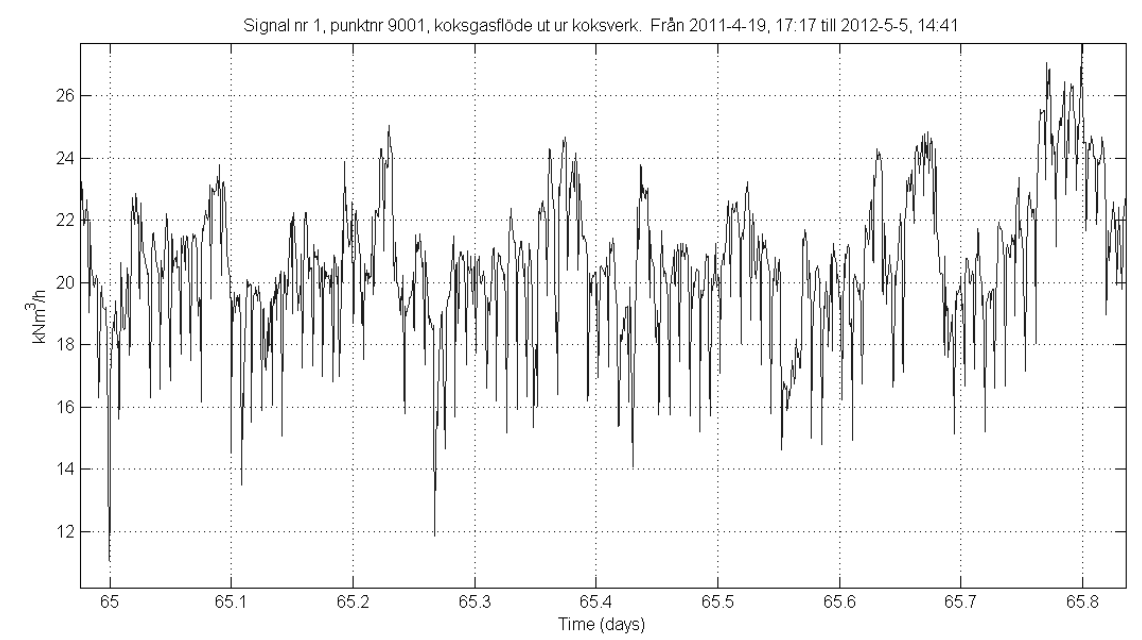

Figure 13: Example of one minute readings of the COG net deliveries (same variable as in Figure 7 but more recent data and higher sampling rate). 
component with period length 207 minutes during the first 145 days of measurement and then increasing up to and staying constant at period length 233 minutes during day $215-383$ of the measurements. The period of 207 minutes $(=0.14$ days) can also be observed in the zoomed in part of the original measurement that is plotted in Figure 13, with one period ranging roughly from 65.1 to 65.23 days.

The continuous wavelet transform could be of interest for future studies. The most important application is probably in the beginning of a study, where it can be used to scan for the most appropriate wavelengths for the following conventional wavelet studies.

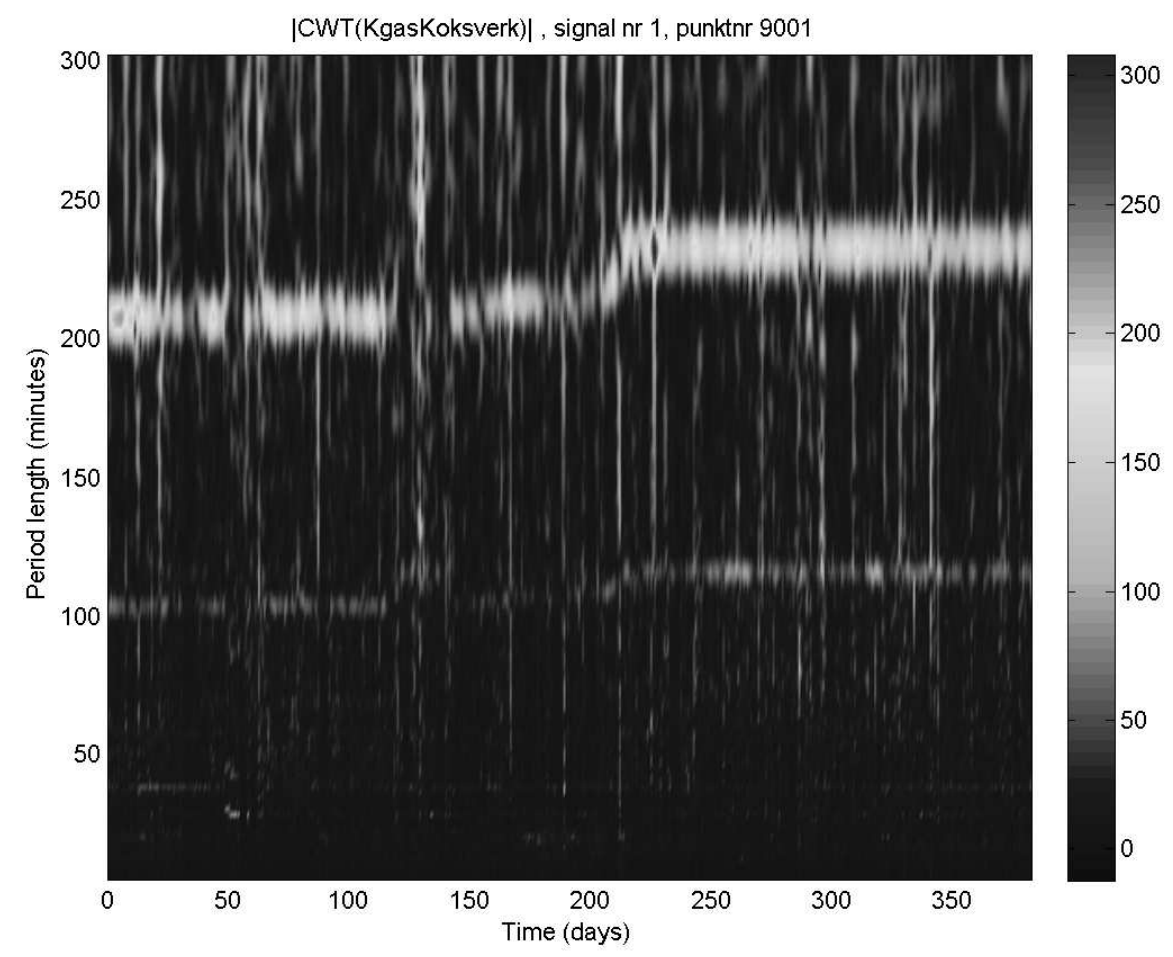

Figure 14: A continuous wavelet transform (WT) is here used for analyzing how the frequency contents changes with time for the COG delivery. A bright horizontal line highlights a dominating oscillation frequency that is much more easy to track throughout the year in this plot than in the zoomed in measurement data in Figure 13. 


\subsection{Wavelet vs Fourier Analysis}

The Fourier analysis uses addition of continuous sinus curves and it can thus be expected to be less suited for fluctuations where frequency, waveform and amplitude are fluctuating with time. Previous studies at SSAB confirmed this problem; however it was possible to get meaningful Fourier results by making the studies over different period lengths: short lengths to study short variations and long periods to catch longer ones. This is OK if it is possible to select period that are representative. The advantage with wavelet analysis is that it can work in one step and cover a long period with variations over time, and it can also handle different types of fluctuations, e.g., spikes. Cf. [9].

\section{Conclusions}

- A study has been made to investigate the fluctuations in the coke oven gas system of an integrated steel plant. Wavelet methodology was used as the fluctuations were expected to have a non-periodic behavior.

- The study shows that there are important gas fluctuations that could influence external use, e.g., for fuel production.

- The study confirmed that the behavior was non periodic. In addition to variations in frequency and amplitudes, there are also different shapes of the fluctuations, e.g. a relatively high ratio of spikes. Different types wavelet tools were tested, and one that showed good behavior with that type of fluctuations was chosen. (Coif 2).

- The fluctuations are of both short and long wavelength.

- Longer wavelengths could probably be parried by good production planning.

- For short wavelengths some kind of safety margin could be appropriate. It could be estimated to around $1.5 \mathrm{kNm}^{3} / \mathrm{h}$.

- The existing buffer capacity in gasholders etc. is too small to cover the fluctuations.

- Good production planning can partly compensate low buffer capacity. An example from a period with gas holder failure is discussed as an example. 
- Some user applications have fluctuations that cause upward spikes in the net availability. These are not harmful but it could be difficult to use the extra energy in them if they are short.

- One objective was to test the wavelet technique for that environment. It showed to be well aimed to analyse the different types of intermittent and irregular variations with different waveforms that are abundant in the steel plant network.

- The present study was carried out for a period for which only data based on hourly means were available. A tentative study based on data with one minute sampling rate pointed out that future studies including shorter wavelengths could be of interest.

\section{Acknowledgement}

We would like to thank the Swedish Energy Agency and the companies SSAB EMEA, Grontmij AB and Nordlight AB for financing this work.

\section{References}

[1] Lundgren J, Asp B, Larsson M, Grip CE. Methanol production at an integrated steel mill. In: Proceedings of the 18th International Congress of Chemical and Process Engineering. Prague, Czech Republic; 2008,WWW: http://pure.ltu.se/portal/files/2334192/Methanol_production_at_an_integrated_steel_mill_FINAL.pdf.

[2] Lundgren J, Grip CE, Ekbom T, Grip N, Hulteberg C, Larsson M, et al. Increased energy efficiency and carbon dioxide -reduction in steel mills - methanol from steel work off-gases. Final project report to the Swedish Energy Agency; Luleå University of Technology; 2012. WWW: http://pure.ltu.se/portal/files/40297431/LGE12.pdf.

[3] Ekbom T, Hulteberg C, Grip CE, Larsson M, Lundgren J, Nilsson L, et al. Methanol production from steel-work off-gases and biomass based synthesis gas. In: Proceedings of the International Conference on Applied Energy (ICAE); vol. 3. Suzhou, China; 2012,WWW: http://pure.ltu.se/portal/files/41370534/A10512.pdf. 
[4] Karlsson M. The MIND method: A decision support for optimization of industrial energy systems - principles and case studies. Applied Energy 2011;88(3):577-89. DOI: 10.1016/j.apenergy.2010.08.021.

[5] Larsson M, Wang C, Dahl J. Development of a method for analysing energy, environmental and economic efficiency for an integrated steel plant. Applied Thermal Engineering 2006;26(13):1353-61. DOI: 10.1016/j.applthermaleng.2005.05.025.

[6] Wang C, Nordgren S, Lindblom B, Savonen S, Hedpalm T, Larsson $\mathrm{M}$, et al. Conceptual design of an integrated heating system at LKAB Malmberget with consideration of social-environmental damage costs. Journal of Cleaner Production 2010;18(9):944-51. DOI: 10.1016/j.jclepro.2010.01.021.

[7] Wang C, Larsson M, Ryman C, Grip CE, Wikstrm JO, Johnsson A, et al. A model on $\mathrm{CO}_{2}$ emission reduction in integrated steelmaking by optimization methods. International Journal of Energy Research 2008;32(12):1092 -106. DOI: 10.1002/er.1447.

[8] Zhu Y, Huang G, Li Y, He L, Zhang X. An interval full-infinite mixedinteger programming method for planning municipal energy systems - a case study of beijing. Applied Energy 2011;88(8):2846-62. DOI: 10.1016/j.apenergy.2011.01.058.

[9] Kong H, Qi E, Li H, Li G, Zhang X. An MILP model for optimization of byproduct gases in the integrated iron and steel plant. Applied Energy 2010;87(7):2156-63. DOI: 10.1016/j.apenergy.2009.11.031.

[10] Bergh J, Ekstedt F, Lindberg M. Wavelets. Lund, Sweden: Studentlitteratur; 1999. ISBN 91-44-00938-0.

[11] Mallat S. A wavelet tour of signal processing — The Sparse Way. Academic Press; third ed.; 2009. ISBN 978-0-12-374370-1.

[12] Grip N. Wavelet and Gabor frames and bases: Approximation, sampling and applications. Doctoral thesis 2002:49; Luleå University of Technology; SE-971 87 Luleå; 2002. WWW: http://epubl.luth.se/14021544/2002/49/index.html. 